\title{
Territoire, culture et représentation : une approche communicationnelle des guides touristiques sur la Suisse
}

Philippe Viallon et Dounya Gaham

\section{(2) OpenEdition \\ Journals \\ Édition électronique \\ URL : http://journals.openedition.org/tourisme/512 \\ DOI : $10.4000 /$ tourisme.512 \\ ISSN : 2492-7503 \\ Éditeur \\ Éditions touristiques européennes}

\section{Édition imprimée}

Date de publication : 1 juin 2011

Pagination : 41-52

ISSN : 2109-5671

\section{Référence électronique}

Philippe Viallon et Dounya Gaham, «Territoire, culture et représentation : une approche communicationnelle des guides touristiques sur la Suisse », Mondes du Tourisme [En ligne], 3 | 2011 mis en ligne le 30 septembre 2015, consulté le 03 mai 2019. URL : http://journals.openedition.org/ tourisme/512 ; DOI : 10.4000/tourisme.512

\section{(c) $(1) \&$}

Mondes du tourisme est mis à disposition selon les termes de la licence Creative Commons Attribution - Pas d'Utilisation Commerciale - Pas de Modification 4.0 International. 


\section{Territoire, culture et représentation :}

\section{une approche communicationnelle}

\section{des guides touristiques sur la Suisse}

\author{
PHILIPPE VIALL ON [philippe.viallon@hispeed.ch] \\ Professeur, université de Strasbourg
}

Résumé. Depuis le numéro 10 de la revue Communications

(1967), consacré au tourisme, on connaît l'importance de l'analyse des guides touristiques pour la compréhension du phénomène du tourisme. Cet article fait tout d'abord le point sur la recherche sur ce support. Puis il évoque le cas de la Suisse qui est particulièrement intéressant, car, si le phénomène touristique est par nature interculturel, le pays a déjà une interculturalité interne entre ses trois zones linguistiques. L'article analyse onze guides sur la Suisse en italien, allemand, français et anglais, et confirme trois hypothèses : chaque guide a tendance à favoriser sa zone de langue-culture aussi bien au niveau quantitatif que qualitatif ; la culture du groupe-émetteur transparaît dans les guides; quelle que soit la culture, des stéréotypes et clichés sont présents.

\author{
DOUNYA GAHAM \\ [dgaham@travelclick.com] \\ Account executive, Travelclick Europe
}

Abstract. The importance of guidebooks for understanding the phenomenon of tourism has been well-understood since the appearance in 1967 of number 10 of the periodical Communications, dedicated to tourism. This article first reviews what research has shown us about the phenomenon. The second half of the article is devoted to Switzerland, chosen as a case study because, if tourism is by definition inter-cultural, tri-lingual Switzerland is, within its own borders, multi-cultural. An analysis of eleven guidebooks on Switzerland in Italian, French, German, and English, confirms three hypotheses: the guidebooks have a tendency, both qualitatively and quantitatively, to favor the regions of the country that are linguistically the same as the language in which the guidebook is written; the guides' authors are not culturally neutral, their own cultural predispositions can be read between the lines; regardless of the cultural origins of the guide, stereotypes and clichés are present in all. 


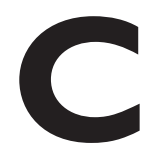

ette contribution est la première étape d'un projet scientifique sur la communication touristique en Suisse, projet soutenu, entre autres par le Fonds national suisse de la recherche scientifique. Elle vise à mettre en place une approche communicationnelle du tourisme dans ce pays et à étudier des phénomènes qui, certes, ont pu être partiellement abordés par d'autres disciplines (par exemple la géographie, la sociologie ou le marketing), mais auxquels l'approche communicationnelle offre de nouvelles perspectives de compréhension des phénomènes sociaux pour le monde académique et des perspectives d'action pour le monde professionnel. Parmi toutes les composantes de la communication, les supports de communication touristique sont nombreux (Boyer et Viallon, 1994, pp. 38-66). Il y a d'abord ceux qui sont émis par les professionnels (affiches, dépliants, catalogues, sites internet...) et ceux qui émanent des touristes eux-mêmes : dans cette dernière catégorie, où longtemps le bouche à oreille a régné seul, la montée en puissance des forums et blogs sur internet est en train de changer la donne. Entre ces deux groupes, il reste la production de professionnels qui n'ont que rarement le tourisme comme activité principale, notamment les médias (presse, radio, télévision, cinéma...) et les éditeurs de guides touristiques.

C'est cette dernière catégorie que nous allons analyser. Le guide touristique a une longue histoire. Elle commence en 1552 avec le Guide des chemins de France, qui fut un succès de la famille des imprimeurs Estienne (Boyer, 1999, p. 58). Plus tard, les jeunes lords anglais, inventeurs du "tour" et par là-même du tourisme, rédigent instructions et recommandations qui sont utilisées par leurs successeurs. Les grands guides naissent au XIX ${ }^{\mathrm{e}}$ siècle, avec les Murray en Angleterre, les Baedeker en Allemagne ou les Joanne en France. Ils sont le produit d'une bourgeoisie qui imite les pratiques de la noblesse. Déjà, les traductions sont fréquentes. $\mathrm{Au} \mathrm{XX}^{\mathrm{e}}$ siècle, la diffusion du tourisme comme pratique sociale, notamment après la Deuxième Guerre mondiale, va encourager dans les grands pays européens la création de collections, qui deviennent des marques : "le" Lonely, "le" Michelin. Chacun a l'ambition de dire ce qu'il faut voir dans un pays ${ }^{(1)}$. On a donc des discours concurrents au sein d'une même langue-culture, mais aussi d'une langue-culture à l'autre à propos d'un même pays. C'est l'objet de cet article que de comparer cette polyphonie de discours sur un pays, en l'occurrence la Suisse ; l'approche s'inscrit aussi dans une dimension interculturelle qui est, dans le cas présent, particulièrement intéressante. En effet, si la rencontre interculturelle est souvent le fondement du tourisme, la Suisse regroupe trois régions linguistiques avec leurs spécificités culturelles : la Suisse alémanique, à tradition germanophone, la Suisse romande, dont les liens avec la culture francophone sont forts, et le Tessin, qui se rapproche de l'Italie. C'est pour cela que le choix a été fait d'analyser des guides issus des trois zones de langue-culture ainsi que de guides de l'édition anglophone, qui pourront servir d'élément de comparaison.

Les questions liées aux guides touristiques sont nombreuses. Depuis, entre autres, les travaux sur le tourisme de Pierre-Jean Thumerelle (1986) et ceux de Mathis Stock (2005), on sait que la mobilité est une des caractéristiques essentielles de l'humanité et du tourisme. Les guides accompagnent souvent cette mobilité. Quelles sont les principales fonctions d'un guide? Si le côté “indications données sur le territoire étranger, sur des pratiques différentes, sur des réalités surprenantes, en un mot sur l'autre" est souvent mis en avant, faut-il pour autant négliger les indications données sur l'auteur, sur le groupe émetteur du guide ? En effet, à l'instar du titre de l'ouvrage de Tzetan Todorov Nous et les autres (1989), le guide n'en dit-il pas autant sur ses auteurs et leurs représentations sociales que sur la réalité qu'il est censé mettre en avant ? Ou bien peut-on aller jusqu'au phénomène décrit par Edward Saïd (1995) lorsqu'une culture, l'Occident, crée un autre culture, l'Orient ? On voit donc l'intérêt qu'il y aurait à oppo- 
ser une production endogène, celle d'un guide suisse sur la Suisse, à des productions partiellement exogènes, des guides français sur la Suisse, ou totalement exogènes, des guides anglais sur la Suisse. À ce propos, il faut constater l'absence d'un guide suisse sur la Suisse, quelle que soit la langue considérée. Les Suisses ne peuvent pas dire dans un guide touristique national et général qui ils sont, ni comment ils se voient ${ }^{(2)}$. Ils doivent s'en remettre à d'autres, ce qui n'est pas sans poser un problème d'identité.

La question des usages des guides est également importante : l'achat d'un guide est-il toujours suivi d'un achat de voyage ? Le guide est-il lu avant le voyage ou emporté avec soi, voire consulté après la pratique touristique pour confirmation? $\mathrm{La}$ question des stratégies de communication des guides est également essentielle : a-t-on affaire à un discours scientifique, vulgarisateur, pédagogique, distrayant ou tout simplement utilitariste ? Et, en conséquence, quelle place donnet-il au lecteur ? Est-ce celle de "l'idiot du voyage", comme l'évoque Jean-Didier Urbain (199|)?

Dans le paysage touristique, le guide occupe une place à part : c'est un discours certes de professionnels, mais pas de ceux du terrain, ce sont des professionnels de l'édition qui le produisent. Quelle est leur marge de manœuvre entre les acteurs touristiques, dont ils ont besoin comme informateurs, et le lecteur, dont ils doivent garder la confiance ? Peut-on encore parler de "guides bleus, guides verts et lunettes roses", comme le faisait Bernard Lerivray (1975) dans les années 1970 dans la lignée des critiques d'un Hans Magnus Enzensberger (1965) ?

Pour résoudre la complexité du support "guide", cet article va d'abord délimiter un cadrage théorique ; ensuite, il comparerera les guides sur la Suisse les plus connus : Michelin, Routard et Petit Futé pour le monde francophone, Reise Know how, Baedeker et Polyglott pour le monde germanophone, Ulysse Moizzi et TCI (Touring club italiano) pour le monde italophone. Les guides anglophones - Fodors, Lonely Planet et DK Eyewitness - ont été ajoutés à l'analyse afin d'observer ce qui se passe lorsqu'il n'y a pas de liens entre la culture "décrivante" et la culture décrite. Deux hypothèses guident ce travail : d'une part, les différents guides ont tendance à favoriser leur propre zone de langue-culture, au niveau tant quantitatif que qualitatif ; d'autre part, il y a des stéréotypes, des clichés communs et récurrents dans tous les guides, quel qu'en soit le pays émetteur. Nous utiliserons une approche quantitative (comptage de pages, de photos, d'articles selon la disposition du guide, classement alphabétique ou par région, etc.) et qualitative (appréciation des formules, présence d'adjectifs mélioratifs ou péjoratifs par rapport au pays qui juge, etc.) sera utilisée. Les éléments communs des guides (indications pratiques, informations générales sur le pays, l'histoire, les transports, etc.) ont été pris en compte à part pour la partie quantitative, puisqu'ils ne peuvent pas être rattachés à l'une ou l'autre région.

\section{LE GUIDE}

Pour cibler la problématique de cet article, le guide va être abordé selon cinq aspects : sa logique, l'espace, le temps, sa critique et son statut en mutation. Ils n'ont pas l'ambition de dire de manière exhaustive ce qu'est un guide touristique, mais de pointer les éléments les plus pertinents pour notre sujet.

\section{Une logique}

Hans Magnus Enzensberger (1965, p. 167) a bien montré que c'est le sight-seeing, ou, pour les amateurs de culture classique, le vivendum, qui est à la base du guide: "La chose à voir ne mérite pas seulement d'être vue, elle exige de l'être. Est à voir ce qu'on est tenu d'avoir vu." Le guide est donc une forme de contrainte sociale qui pèse sur le touriste. Ce discours d'autorité, caractéristique des guides du XX $X^{e}$ siècle, s'est certes atténué : du Guide bleu d'Hachette au Guide $d u$ routard en passant par le Guide vert Michelin, on note dans les guides français l'évolution vers une approche différente. Mais si la 
forme change, le principe de discours de référence reste, car il fait la force du guide. Le touriste potentiel, quittant son monde, entre dans un autre monde, celui du guide, avant de pénétrer dans celui qu'il veut découvrir. C'est un entre-deux, avec des règles, des formes précises. Une des caractéristiques les plus frappantes est sa non-scientificité ; le guide poursuit la tradition de la compilation, c'est une anti-thèse de doctorat : il ne donne pas ses sources, rarement de bibliographie, affirme sans justifier. Sa volonté permanente de diviser, classer le monde en catégories est également remarquable. Il donne au monde une rationalité qu'il n'a sans doute pas, il propose une lecture de réalités étrangères adaptée à son public et utilise un langage spécifique : des étoiles ou tout autre système signifiant, comme par exemple la remarque ambiguë "vaut le détour" ou celle du Routard, plus paradoxale, "à éviter absolument, car rempli de touristes".

En résumé, le guide est un des gardiens du temple, une aide à la reproduction de performances normées, un produit éminent d'une culture à propos d'une autre culture.

\section{Un espace}

Un guide, c'est d'abord un espace nommé et désigné par un titre. La très grande majorité des collections de guides donne un nom de pays ou de zone géographique à leurs ouvrages : cela facilite le choix dans la série. Car un guide, avant d'être un livre sur la Suisse, s'inscrit d'abord dans une collection, celle de son éditeur, et le Guide du routard Suisse, avant d'être un ouvrage unique, est à mettre en regard avec le Guide du routard Allemagne et celui sur l'Italie, etc. La renommée des collections est essentielle dans les décisions d'achat ; le touriste achètera le "routard" de la Suisse parce qu'il a apprécié celui de l'Italie. Cet espace renvoie donc à deux types de logique qui s'entrecroisent : la logique nationale des réalités décrites, qui sont particulièrement complexes dans le cas de la Suisse avec ses quatre langues nationales et ses trois zones linguistiques, comme cela a été indiqué ; mais aussi la logique des émetteurs : le Guide du routard renvoie à la France, même s'il concerne également les autres lecteurs francophones potentiels; de même, les guides italiens renvoient à l'Italie et les guides allemands à l'Allemagne. Le cas des guides en anglais est plus complexe à cause de la diversité de l'espace anglophone natif, à laquelle s'ajoute le fait que de nombreux touristes pratiquent l'anglais comme langue de communication. Cette conjonction entre ces espaces analysants et ces espaces analysés est d'autant plus intéressante qu'il existe une certaine proximité géographique. Pour dire les choses simplement, un Romand lisant le Guide du routard sur la Suisse aura-t-il le même regard qu'un Français, un Allemand lisant le Baedeker sur la Suisse aura-t-il le même regard qu'un Alémanique ? Une étude de réception des guides, qui ne sera pas faite dans le cadre de cet article, devrait pouvoir répondre à une telle question.

Un autre élément caractéristique des guides est l'espace évoqué. C'est une carte faite de beaucoup de vides, ces espaces que l'on va parcourir sans s'arrêter, pour aller d'un centre d'intérêt à un autre. Est-ce que le mapping est le même d'un guide à l'autre ? L'un des objectifs de cette recherche est aussi de pointer ces différences dans la lecture du territoire étranger. Les vides correspondent donc aux éléments non normés par les guides. L'observation de l'espace touristique se fait, dans le cas des guides étrangers, à partir d'autres espaces, et c'est sur les effets de cette variable que porte notre article.

Dernier élément de l'espace : celui que réservent les guides aux différents lieux choisis. À partir du moment où un lieu est traité, estil abordé avec la même importance dans tous les guides ? L'analyse du rapport entre l'espace décrit et l'espace "discursif papier" (Viallon, 2004, pp. 191-214) est l'un des éléments importants de la comparaison entre guides.

\section{Une temporalité}

Le guide s'inscrit aussi dans une temporalité, celle de son utilisateur. À la suite d'autres chercheurs, nous avons montré avec Marc Boyer (1994) que le touriste vit trois temps 
du voyage : le voyage rêvé, le voyage vécu et le voyage prolongé. Le guide accompagne chacun de ces moments, mais de manière différente.

Dans le voyage rêvé, le guide fait fonction de catalyseur. Avec d'autres supports professionnels (comme les catalogues et les messages publicitaires -, mais aussi des supports qui n'ont rien à voir avec le tourisme (comme les revues, les documentaires télévisuels, voire les films de long métrage), il permet un voyage facile. Pour un prix encore plus bas que celui d'un billet d'une compagnie aérienne low cost, le guide fait voyager son lecteur, dont l'imagination se nourrit de descriptions qui produisent des représentations largement formatées. Combien d'achats d'un guide ne sont jamais suivis de voyage ? Cela renvoie à la complexité de la psychologie et du comportement des touristes (Cousin et Réau, 2009). Comme tout produit soumis aux lois du marché, le guide doit répondre de manière différenciée aux besoins de ses lecteurs : les facteurs push et pull mis en avant dans la littérature anglo-saxonne (Kim et Yoon, 2003, pp. I-22 ; Baloglu et Mc Cleary, 1999, pp. 868-897 ; Fackeye et Crompton, 199I, pp. I016) sur la nature du tourisme sont une aide précieuse pour saisir les différents rôles que peuvent jouer les guides auprès de leurs lecteurs.

Dans le voyage vécu, c'est la fonction pratique qui domine. Le guide est utilitaire et cela explique ses aspects de forme (poids, format, épaisseur) et de fond (quantité et type d'informations offertes). Cette caractéristique est relayée par une dimension économique : un guide efficace est un guide régulièrement actualisé. Pour le touriste, il faut donc le remplacer souvent, pour le plus grand intérêt des éditeurs.

Dans le voyage prolongé, le guide est beaucoup plus utilisé qu'on pourrait le supposer. Déjà dans le train ou l'avion de retour, il est relu pour aider à la mémorisation de noms souvent compliqués pour le touriste ; à la maison, il est utilisé pour classer les photos. Bref, c'est un aide-mémoire, mais aussi une preuve du voyage, à condition qu'il porte les traces de son usage, au même titre que les souvenirs rapportés. Certains éditeurs anticipent même une co-construction du message, qui n'est pas sans rappeler celle d'Umberto Eco (1985), en laissant des pages blanches pour des notes. Le guide est aussi ressorti lors de discussions avec des amis ou prêté dans le cadre d'une recommandation par le bouche-à-oreille, et il recommence ainsi un nouveau cycle.

On le voit, l'appropriation du guide comme support de communication peut se faire à des moments très divers du voyage.

\section{La critique des guides}

Dans les années 1970, le tourisme a été accusé de tous les maux : de Veblen (1899), que l'on a (re)découvert à cette époque, à
Baudrillard (1981), en passant par Enzensberger (1965), Barthes (1970) ou Krippendorf (1975). Ces critiques voyaient dans le tourisme une "soupape pour des individus aliénés par la société", un "ballon d'oxygène de survie pour des populations locales exploitées par le capitalisme mondial" ; il était la cause de l'apparition de constructions hideuses sur des sites idylliques, au point que, selon Georges Cazes (1976), "l'appareil du tourisme international interdit de façon organique toute relation réelle entre visiteur et visité", en un mot, il serait l'un des grands maux de notre société. Le guide était la matérialisation de cette pratique et le plus grand vecteur de l'idéologie bourgeoise (Gritti, 1967, pp. 5 I-64). Depuis les travaux de Jean-Didier Urbain (199|) en France, ceux de Kurt Luger (2000) en Autriche, de Jost Krippendorf (1975) en Suisse ou de Dean MacCannell (1976) pour le monde anglo-saxon, le tourisme est abordé de manière beaucoup plus nuancée. On constate une volonté de voir le tourisme, pas seulement comme un élément $\mathrm{du}$ PNB, mais aussi comme un lieu d'analyse de pratiques sociales complexes et mouvantes. Quelles en sont les conséquences pour le guide ? La critique du tourisme n'a pas empêché cette pratique sociale de connaître une extension remarquable, puisque le nombre de touristes au niveau mondial a plus que doublé durant les vingt dernières années pour atteindre près de 900 
millions en $2010^{(3)}$. Si les grands éditeurs de guides mentionnés ne donnent aucun chiffre, le monde de l'édition a l'habitude de considérer les grandes collections de guides citées plus haut comme des "vaches à lait particulièrement rentables". Il est probable que les ventes ont crû avec le nombre de touristes. Dans une situation aussi confortable, les guides ont donc peu évolué ; seul leur ton a changé. L'exemple le plus caractéristique est celui du Guide du routard, qui considère le lecteur comme un copain, ou encore du guide Lonely Planet, qui confie chaque numéro à un auteur et à toute sa subjectivité. En résumé, ces guides semblent être aujourd'hui considérés comme un mal nécessaire, comme le dit Ariane Devanthéry (2008) dans son article "À la défense de mal aimés souvent bien utiles : les guides de voyage". Mais la situation risque de changer.

\section{Un modèle en évolution}

On ne peut terminer ce rapide point sur les guides sans mentionner les technologies d'information et de communication (Tic); elles ne sont plus nouvelles, mais les pratiques qui en découlent s'inventent encore jour après jour. Que fautil retenir ?

La disparition du support papier en général, et du guide en particulier, n'a pas eu lieu et n'aura pas lieu. Devant la montée en puissance d'internet, chaque ancien média cherche à affirmer ou à réinventer sa spécificité, à trouver un business model compatible avec les nouvelles conditions. Les éditeurs, assez réticents au début pour mettre gratuitement en ligne des informations, ont compris qu'ils pouvaient compenser des éventuelles pertes de revenus de vente par de la publicité en ligne. Si le modèle économique n'est pas encore stabilisé, tout laisse à penser que c'est une question de temps. Pour le moment, rien ne remplace le guide papier, même si les choses peuvent changer. Les inspecteurs des guides ont actuellement comme consigne de lier les informations qu'ils récoltent à des références GPS. Si la première conséquence peut être une aide pour retrouver tel ou tel lieu, on envisage aussi des systèmes dits "pro-actifs" qui feraient au touriste des propositions de visite ou de services à partir de sa position. L'histoire des médias montre que les évolutions dans le domaine culturel se font beaucoup plus lentement que dans la technique : ce n'est sans doute pas pour demain, mais pour après-demain.

Un autre élément lié aux Tic est donné par l'émergence de nouveaux guides. Le temps est fini où les maisons d'édition étaient les seules à pouvoir produire des informations pour les touristes. Chaque individu équipé d'un ordinateur et d'une liaison internet peut, dans un $b l o g$ ou un forum, raconter son voyage, photos et remarques précises qui parlent au lecteur à l'appui, en un mot, tout le monde peut devenir guide. Cette évolution renvoie à cette fameuse une du Time en 2009: "The man of the year is you." Les éditeurs ont compris le danger et ils ont ajouté un forum à leurs sites, ils diffusent des informations qu'ils retenaient autrefois, ils donnent la possibilité de noter les prestations des hôtels, des restaurants. De manière générale, le web 2.0 est générateur de nouvelles pratiques de diffusion d'informations touristiques. Si la technique est nouvelle, la pratique est ancienne : le bouche à oreille est un des plus anciens moyens de diffusion de l'information touristique, même si c'est sans doute celui qui a été le moins étudié.

Les différents aspects du guide envisagés ont montré l'importance d'un tel support de communication dans les représentations de l'autre et l'intérêt d'analyser sa production dans un monde dit globalisé, mais où les cultures locales restent les systèmes interprétatifs dominants de l'altérité, moteur essentiel du tourisme.

\section{LES GUIDES SUR LA SUISSE}

À partir du postulat qu'aucun discours n'est dénué de subjectivité et que, par conséquent, les guides devraient laisser des traces de cette subjectivité, ce travail, mené sur les douze guides les plus courants rédigés en français, allemand, italien et anglais sur la Suisse, a d'abord analysé la place réservée à chaque région linguistique ${ }^{(4)}$. 


\section{Traitement général des régions} dans l'ensemble des guides

Le tableau 1 montre clairement que la Suisse alémanique est moins bien traitée que les autres régions dans l'ensemble des guides. Alors que, aussi bien du point de vue du territoire que de la population, elle représente environ les deux tiers du pays, elle n'a droit qu'à un peu plus de la moitié des pages des guides. La Romandie est légèrement en retrait, mais la différence est faible. Logiquement, c'est le Tessin qui bénéficie de ce traitement. Comment expliquer cette tendance générale très nette ? On pourrait comparer ces chiffres avec les pratiques. Les guides, en bons professionnels de la communication, adapteraient leur discours aux attentes de leur public : celui-ci apprécierait particulièrement le Tessin en s'y rendant fréquemment ; les éditeurs survaloriseraient donc cette région. Mais ce n'est pas le cas. Les nuits d'hôtel, qui sont comptés de manière fiable en Suisse, révèlent qu'en 2008, 64 \% des nuitées ont lieu en Suisse alémanique, $29 \%$ en Romandie et $7 \%$ dans le $\operatorname{Tessin}^{(5)}$. On est très proche des chiffres relatifs au territoire. On pourrait aussi penser que les guides, tous étrangers à la Suisse, refléteraient une image de la Suisse spécifique aux étrangers. Mais, là encore, les chiffres des nuitées dans le Tessin $^{(6)}$ montrent que la proportion d'étrangers y est à peu près la même que dans le reste du territoire (un peu plus de la moitié).

Tableau | • Traitement général des régions dans l'ensemble des guides

\begin{tabular}{|c|c|c|c|c|}
\hline & $\begin{array}{l}\text { Suisse } \\
\text { alémanique }\end{array}$ & $\begin{array}{l}\text { Suisse } \\
\text { romande }\end{array}$ & Tessin & Généralités \\
\hline Ensemble des guides toutes pages confondues & $51 \%$ & $25 \%$ & $15 \%$ & $8 \%$ \\
\hline Sans les généralités & $55 \%$ & $27 \%$ & $16 \%$ & - \\
\hline Territoires $(1)$ & $64 \%$ & $29 \%$ & $7 \%$ & \\
\hline $\begin{array}{l}\text { Population parlant la langue dominante } \\
\text { de chaque région }\end{array}$ & $63 \%$ & $20 \%$ & $4 \%$ & $\begin{array}{l}10 \% \\
\text { (étrangers) }\end{array}$ \\
\hline Sans les étrangers ${ }^{(2)}$ & $70 \%$ & $25 \%$ & $6 \%$ & - \\
\hline
\end{tabular}

(I) Par souci de simplification, on n'a pas envisagé le cas du romanche, dont les locuteurs maitrisent toujours une autre langue nationale. On a également considéré tous les cantons comme monolingues, même si la réalité est plus complexe. Les chiffres sont issus du site de l'Office fédéral de la statistique. (À partir de www.bfs.admin.ch/bfs/portal/fr/index.html [Consulté le ler janvier 20/0].)

(2) Ibid.

Figure | - Le traitement des trois régions selon les quatre langues cultures

Guides francophones

Partie alémanique $\cdot 43,3 \%$

Partie romande $\cdot 35,3 \%$

Tessin $• 13,8 \%$

Généralités • 7,6\%

Guides italophones

Partie alémanique $• 52,5 \%$

Partie romande $\cdot 21,5 \%$

Tessin • 13,5\%

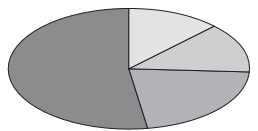

Généralités • 12,5\%

Reste donc le fait que les pratiques touristiques ne correspondent pas aux choix des guides. Deux explications sont dès lors possibles : soit il s'agit d'une erreur stratégique des guides, mais comment expliquer que tous les guides fassent la même erreur ? soit, et cela paraît plus probable, les guides ont compris que le Tessin est une région qui fait rêver, ce qui renvoie à une des fonctions du guide évoquées plus haut. Pour beaucoup, cette région
Guides germanophones

Partie alémanique • 55,6\%

Partie romande • 17,0\%

Tessin $• 19,1 \%$

Généralités • 8,3\%

Guides anglophones (groupe témoin)

Partie alémanique • 54,0\%

Partie romande $\cdot 26,6 \%$

Tessin $• 12,1 \%$

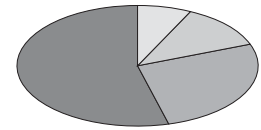

Généralités • 7,3\%

est le début du Sud, mythe relayé par de nombreuses sources, notamment la littérature - "Kennst Du das Land, wo die Zitronen blühen..." écrivait Goethe dans Mignon, en 1795 [Connais-tu le pays où fleurissent les citronniers ?]). Les éditeurs ont compris que le touriste potentiel lira avec plaisir les descriptions du Tessin dans les guides, qu'il donnera ainsi libre cours à son héliophilie, à sa "sudophilie", mais qu'il ne fera 
pas obligatoirement le voyage, par manque de temps ou par éloignement de la région.

Le traitement des trois régions selon les quatre langues-cultures

Si l'on regroupe les guides par zone culturelle d'émission, quelles sont les caractéristiques qui ressortent ( $c f$. figure 1) ? C'est dans les guides allemands que la Suisse alémanique est le plus longuement traitée, mais les guides anglophones ne sont pas loin et les italophones non plus. En revanche, il est clair qu'elle est proportionnellement moins importante dans les guides francophones. Difficulté supposée de la langue, éloignement de la zone géographique francophone, culture germanophone moins "à la mode”, les raisons peuvent être nombreuses et ne peuvent être analysées dans le cadre de cet article.

Les guides francophones donnent de loin la meilleure place à la Romandie, suivis de loin par les anglophones, de plus loin par les italophones et encore plus loin les germanophones. Tout se passe comme si ce relatif désintérêt des francophones envers la Suisse alémanique répondait à celui des germanophones envers la Romandie. L'explication n'est sans doute pas à chercher dans une pseudo-opposition de culture, mais dans des raisons pratiques. Les deux principales parties de la Suisse, la germanophone et la francophone, offrent des formes de tourisme comparables : tourisme de ville, de campagne, de lac, de montagne ; d'été et d'hiver...). Rester dans sa zone linguistique permet de raccourcir les temps de voyage, de faciliter le contact avec la population locale. Les chiffres relatifs à l'origine des touristes pour les nuitées dans les différents cantons de Suisse confirment cette préférence de séjour dans sa propre langueculture $^{(7)}$.

Les guides italophones s'inscrivent dans la même logique : eux aussi évoquent la région proche, le Tessin, de manière privilégiée. Mais cet intérêt n'est pas majeur : avec les guides francophones et anglophones, ils se situent dans une même fourchette étroite. Le point original est que les guides allemands sont encore plus intéressés par cette région que les italophones, au point qu'ils lui accordent plus de place qu'à la Suisse romande.

Enfin les guides anglophones, qui peuvent être utilisés aussi bien par des native speakers aux cultures déjà bien différentes que par des étrangers qui pratiquent l'anglais comme langue de communication, sont les plus proches des chiffres de la population et de la superficie du territoire, tout en montrant une surreprésentation du Tessin. Les résultats de l'analyse quantitative confirment donc l'hypothèse d'une meilleure représentation de la zone culturellement et linguistiquement proche en ajoutant un résultat inattendu, la prééminence du Tessin, prééminence d'autant plus étrange qu'elle n'est pas confirmée par la réalité des pratiques touristiques, comme cela a été démontré.

\section{Analyse qualitative}

Une analyse qualitative a été menée sur l'ensemble des guides, y compris sur la partie "généralités”, car c'est souvent là que les marques de l'émetteur sont les plus nombreuses. Les guides germanophones restent relativement neutres, sauf sur certains sujets plus sensibles comme la langue. On lit dans Reise Know How : "Wenn es für Schweizer recht schwierig ist, sich in geschliffenem Hochdeutsch zu unterhalten [...]" [Comme il est bien difficile pour des Suisses de discuter en haut allemand de qualité] (p. 92). Des Suisses alémaniques liront de telles lignes avec un sentiment mitigé. Le Baedeker est plus concret en évoquant la diversité linguistique, source aussi de problèmes qui débordent de la seule sphère linguistique : "Das Schwyzerdütsch, das aus dem Alemmanischen hervorging und sich in eine grosse Zahl von Mundarten gliedert, ist ein wichtiger Aufhänger für innerschweizerische Ressentiments" [Le suisse allemand, qui vient de l'alémanique et qui est composé d'un grand nombre de dialectes, est un point important pour les ressentiments internes à la Suisse] (p. 29). Polyglott n'entre pas dans ce genre de considérations et se limite aux informations pratiques.

Les guides francophones, qui 
sont en fait tous français, font tous référence à la culture française, mais pas de la même manière. Le Guide du routard le fait très fréquemment : "On peut finalement séjourner en Suisse [...] comme dans la province française” (p. 35) ou "La cuisine romande est inspirée, certains disent calquée sur la cuisine française" (p. 37). L'expression est souvent condescendante. Il est probable que, là aussi, des Suisses romands seront irrités par le chauvinisme fréquent du ton et confortés dans leurs clichés sur les Français ayant une haute idée d'eux-mêmes. Le Petit Futé fait également de nombreux rapprochements, mais qui ont moins ce ton "donneur de leçons". L’objectif est plus explicatif, pédagogique: "La Suisse bénéficie d'un système de santé à la française" (p. 16) ou "Il n'est pas sûr que les Suisses soient davantage assoiffés de culture que les Français, mais [...]” (p. 17). Le Guide Michelin, pour sa part, adopte un style beaucoup plus neutre, ne se permet pas de jugements, si ce n'est dans sa fonction première, celle de classer les curiosités. La relation avec la France est quasi absente, sauf dans les indications pratiques pour le voyage.

Pour les guides italophones, la culture d'origine est clairement affichée dans l'Ulysse Moizzi : "I ciclomotori che in Italia non hanno targa [...] se provvisti delcontrassegno ufficiale ' $I$ '” [Les scooters qui n'ont pas de plaque en Italie
(...) s'ils ont le signe officiel "I"] (p. 42) ou "più all'italiana nel canton Ticino" [plus à l'italien dans le canton du Tessin] (p. 53). Le TCI reste sobre dans ses évocations de l'Italie: "Questa nuova edizione della guida [...] anche gli aspetti maggiormente vicini alla sensibilità del turista italiano" [cette nouvelle édition du guide (...) les aspects les plus proches de la sensibilité du touriste italien] (p. 3). Les guides anglais enfin ont des stratégies diverses. Fodors renvoie fréquemment à la culture nord-américaine : "If you are looking for an American-style chain motel comfort..." [Si vous cherchez le confort du type de celui d'un motel américain] (p. 52) ; "The entire state of Switzerland is smaller than the state of West Virginia" [La Suisse tout entière est plus petite que l'État de la Virginie-Occidentale] (p. 34). Lonely Planet aborde la question avec une subjectivité qui renvoie plus à l'auteur qu'à sa nationalité : "Travellers from North America will find all Europe more expensive, and the pain in Switzerland only marginally worse" [Les voyageurs Nord-Américains trouveront toute l'Europe plus chère ; ce ne sera que marginalement plus douloureux en Suisse] (p. 9). DK Eyewitness n'établit aucune relation avec un pays anglo-saxon, si ce n'est dans les rubriques pratiques.

Ces quelques indications de nature qualitative sur le rapport entre la langue-culture d'origine et la Suisse en général ou une de ses régions reflètent la tendance de l'analyse du discours et mettent en relief une tendance nette : d'un point de vue général, la culture du pays émetteur se retrouve dans tous les guides, notamment dans les rubriques utilitaires. Mais elle se manifeste de manière différente selon la collection (par exemple, la renommée pour le Guide Michelin ou le ton chauvin pour le Guide du routard). Une généralisation entre des guides issus d'une même zone de langue-culture s'avère difficile en raison des énormes différences existant d'une collection à l'autre. On a l'impression que, par delà le rapport privilégié entre langues-cultures, c'est la stratégie choisie par le guide qui est décisive. L'hypothèse est donc globalement confirmée par l'analyse qualitative, mais l'importance des stratégies discursives spécifiques à chaque collection est grande.

\section{LES STÉRÉOTYPES COMMUNS}

La deuxième hypothèse postule que, quelle que soit la langue-culture des guides, il existe un certain nombre de stéréotypes ou clichés communs. Les travaux sur les stéréotypes, notamment dans le cadre de la communication interculturelle, ont apporté depuis vingt ans un ensemble d'informations intéressantes, notamment avec Ruth Amossy et Anne Herschberg-Pierrot (1997) ou Steele (1999) dans le monde anglo-saxon, qui ont montré la nécessité des stéréotypes. Ils sont 
Figure $2 \bullet$ Classement des stéréotypes par fréquence d'apparition

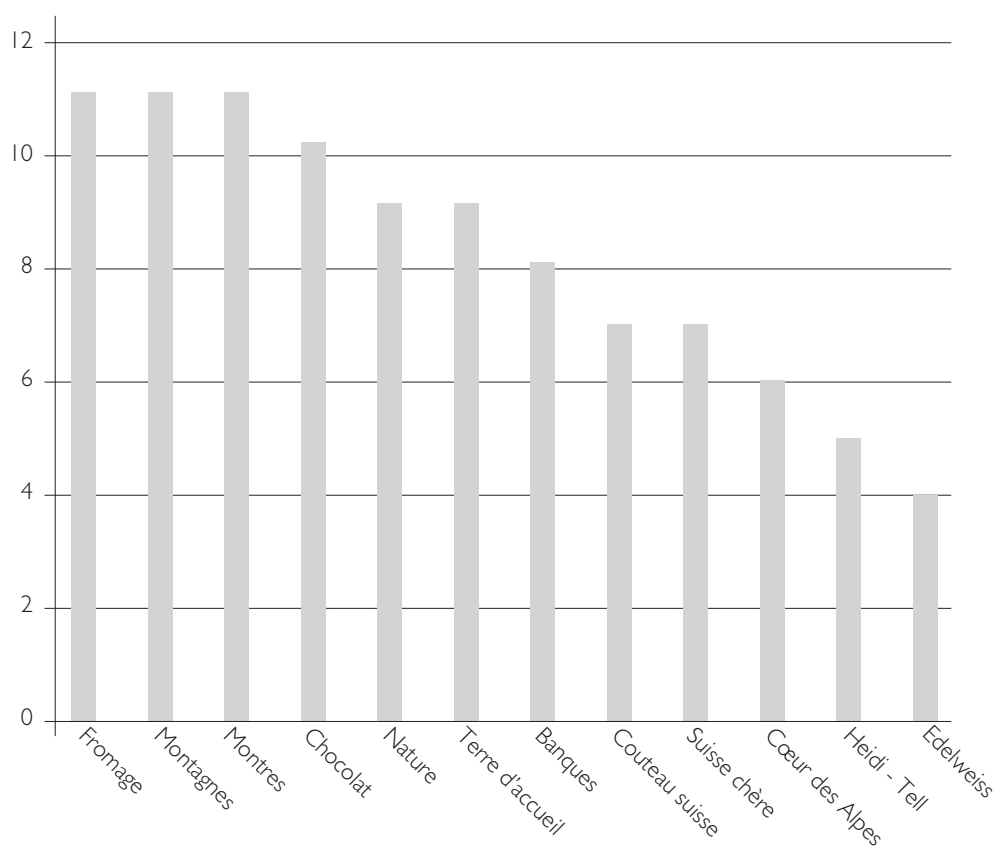

Figure $3 \bullet$ Classement des guides par nombre de stéréotypes

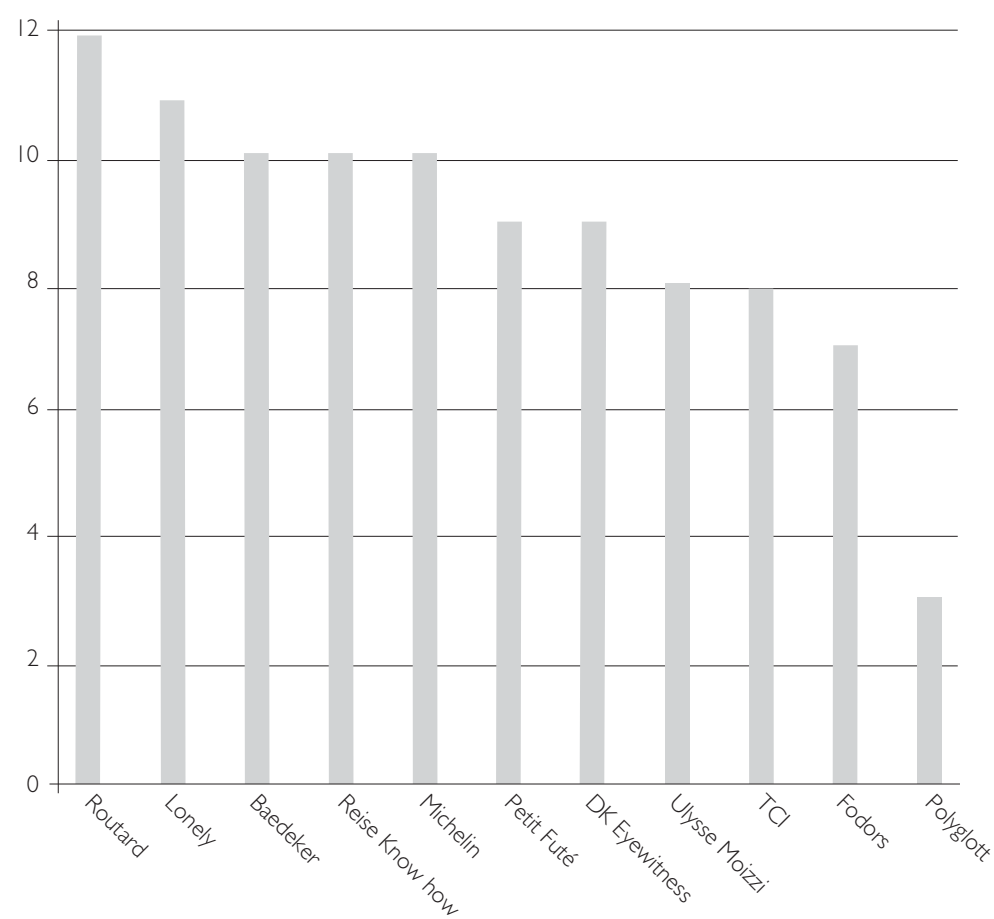

une étape nécessaire à la construction d'une représentation sociale. Pour utiliser une métaphore, on ne peut construire sur du vide et une représentation, même erronée, vaut mieux que rien. Autre élément important : si certains stéréotypes sont négatifs, d'autres sont positifs. Il faut donc avoir un rapport beaucoup plus serein aux stéréotypes. Pour cette étude, nous nous sommes cependant heurtés à un problème heuristique : comment repérer un stéréotype ? Dire que la Suisse est le pays des montres ou du chocolat, est-ce un stéréotype ou cela correspond-il à la réalité ? Ce sont des branches importantes de l'industrie suisse, et il est difficile de ne pas trouver d'évocation de la Suisse sans les montres. Une solution pragmatique a été trouvée : est considéré comme stéréotype tout ce qui est mentionné par les auteurs comme tel ou désigné par des quasi-synonymes ${ }^{(8)}$. Cela a conduit à une liste de douze stéréotypes récurrents communs à l'ensemble des guides (cf. figure 2).

Le premier enseignement que l'on peut tirer de cette liste est qu'elle n'apporte pas beaucoup de surprises $^{(9)}$ : à part peut-être "Suisse, terre d'accueil", ces représentations de la Suisse sont largement partagées, comme s'il y avait une doxa interculturelle sur la Suisse. Mais, si tous les guides s'entendent pour évoquer fromages, montres et montagnes, ils n'activent pas systématiquement tous les stéréotypes. Ceux qui se réfèrent à l'edelweiss 
et aux personnages mythiques comme Heidi ou Tell, par exemple, semblent en perte de vitesse.

Le classement des guides selon le nombre de stéréotypes qu'ils reprennent ( $c f$. figure 3) est intéressant : d'abord, il ressort que même les guides qui se veulent objectifs par leur ton, leur manière de traiter le sujet (Michelin, Baedeker) recourent eux aussi aux stéréotypes. Ensuite, le Guide du routard et le guide Lonely Planet sont ceux qui véhiculent le plus grand nombre de stéréotypes : cela confirme le côté "personnalisation" évoqué plus haut à propos de l'analyse qualitative. Enfin, il n'est pas possible de faire un classement par langue-culture. Là encore, la stratégie éditoriale semble plus forte que la variable nationale. L'hypothèse selon laquelle tous les guides ont recours aux stéréotypes est donc vérifiée, mais l'analyse montre que les différences d'intensité de leur usage tiennent davantage de l'ordre de la stratégie éditoriale que de la langue-culture des guides.

\section{CONCLUSION}

Ce travail sur les guides touristiques sur la Suisse a montré que l'approche communicative permet de mettre dans une perspective originale le support touristique, dans ce cas, le guide. Un guide touristique s'inscrit d'abord dans une logique de stratégie éditoriale au sein d'une maison d'édition avec une réputa- tion, dans le cadre d'une collection avec une image de marque. Le ton est la marque de fabrique du Guide $d u$ routard, qui se retrouve dans tous les exemplaires de la collection, l'originalité de chaque auteur est le fil rouge du guide Lonely Planet, le classement apparemment objectif et neutre est la caractéristique du Guide Michelin, DK Eyewitness est fortement illustré, etc. Cet élément de marketing exerce une influence non négligeable sur la façon dont les guides évoquent la Suisse. Analyser onze guides issus de quatre langues-cultures a permis à la fois de montrer des éléments communs à tous, une sorte d'image internationale de la Suisse touristique, mais aussi de mettre en évidence la priorité que des pays ayant une langue-culture proche de la Suisse accordent à telle ou telle région. C'est la confirmation que tout texte dit autant sur la réalité représentée que sur son auteur.

Il faut enfin insister sur le fait que les éditeurs de guides nationaux forment, au niveau mondial, un club fermé : trois langues (anglais, français, allemand) produisent la quasi-totalité des guides. Tous les autres sont des traductions ! Ce sont au total à peine une douzaine d'éditeurs issus des grandes cultures occidentales qui disent le monde. Quant à la Suisse, on ne peut que déplorer qu'elle ne produise plus de guides sur elle-même : être dépendant de ses voisins, avec qui les rapports sont pour le moins complexes, pour signifier son iden- tité touristique ne va pas sans poser problème. La montée en puissance des "nouveaux" modes d'information touristique, permettant plus de personnalisation, devrait pouvoir modifier cette donnée.

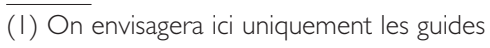
nationaux et non thématiques.

(2) Un ouvrage a existé, publié en français dans la série des guides Olizane à la fin du $X X^{e}$ siècle. Si cette série reste active, elle a renoncé, pour des raisons commerciales, à rééditer celui qui est consacré à la Suisse.

(3) Chiffres de l'Organisation mondiale du tourisme. (À partir de http://www.unwto.org/ facts/eng/pdf/highlights/UNWTO_Highlights09 _fr_LR.pdf [Consulté le Ier janvier 20 I0].)

(4) Le décompte s'est fait selon l'espace occupé (nombre de pages et, si nécessaire, demi ou quart de page)

(5) Par souci de simplification, on n'a pas envisagé le cas du romanche, dont les locuteurs maîtrisent toujours une autre langue nationale. On a également considéré tous les cantons comme monolingues, même si la réalité est plus complexe. Les chiffres sont issus du site de l'Office fédéral de la statistique. (À partir de www.bfs.admin.ch/bfs/ portal/fr/index.html [Consulté le ler janvier 2010].)

(6) Ibid.

$\overline{\text { (7) } \mathrm{lbid} \text {. }}$

(8) Les différences qui existent entre stéréotypes, clichés, images, lieux communs, idées reçues... ne sont pas pertinentes pour ce qui concerne cette analyse.

(9) Par rapport notamment aux nombreuses études et enquêtes menées à l'étranger sur l'image de la Suisse, en général ou touristique, par la société suisse Image [http://unww.imageschweiz.ch]. 
RÉFÉRENCES BIBLIOGRAPHIQUES

Ruth Amossy et Anne Herschberg-Pierrot, Stéréotypes et Clichés, Nathan, 1997.

Seyhmus Baloglu et Ken McCleary, "A Model of Destination Image Formation", Annals of Tourism Research, 26, 1999.

Roland BARTHES, Mythologies, Seuil, 1970.

Jean BAUdRILlARD, Simulacres et simulation, Galilée, 1981.

Marc BOYER et Philippe VIALLON, La Communication touristique, Puf, 1994.

Marc BOYer, Le Tourisme de l'an 2000, Pul, 1999.

Georges CAZES, Le Tiers-Monde vu par les publicités touristiques: une image mystifiante, Centre des hautes études touristiques,

1976.

Saskia CousIN et Bertrand RÉAU, Sociologie du tourisme,

La Découverte, 2009.

Ariane DEVANTHÉRY, "À la défense de mal-aimés souvent bien utiles : les guides de voyage", Articulo, 2008 [en ligne]. À partir de http://articulo.revues.org/index747.html

[Consulté le ler janvier 2010].

Umberto Eco, Lector in fabula, Grasset, 1985.

Hans Magnus ENZENSBERGER, Culture ou mise en condition ?

Les Lettres nouvelles, 1965.

Peter C. FACKEYE et John L. CROMPTON, "Image Differences between Prospective, First-Time and Repeat Visitors to the Lower Rio Grande Valley", Journal of Travel Research, 30, 2, 1991.

Jules GrITTI, "Les contenus culturels du Guide bleu”,

Communications, 10, 1967.

Seehyung KIM et Yooshik Yoon, "The Hierarchical Effects of Affective and Cognitive Components on Tourism Destination Image", Joumal of Travel and Tourism Marketing, 14, 2, 2003.

Jost KRIPPENDORF, Les Dévoreurs de paysages,

Éditions 24 heures, 1975.

Bernard LerIVRAY, Guides bleus, guides verts et lunettes roses, Cerf, 1975.

Kurt Luger, "Kommunikation im Tourismus", Aurora, 2000

[en ligne]. À partir de http://www.aurora-magazin.at/

gesellschaft/inhalt_gesellschaft.htm

[Consulté le Ier janvier 20I0I]
Dean MacCannelL, The Tourist : a New Theory of the Leisure

Class, Schocken books, 1976.

Edward W. SAIID, Orientalism, Penguin books, 1995.

Claude M. Steele, "Thin Ice: Stereotype Threat and Black

College Students", Atlantic Magazine, 1999 [en ligne].

À partir de http://www.goalconsulting.org/page5/files/

Stereotype\%20Threat\%20Steele.pdf

[Consulté le I er janvier 2010].

Mathis StOCK, "Les sociétés à individus mobiles : vers un nouveau mode d'habiter? L'exemple des pratiques touristiques", EspacesTemps.net, Textuel, 2005 [en ligne]. À partir de http://www.espacestemps.net/document | 353.html [Consulté le I er janvier 2010].

Pierre-Jean Thumerelle, Peuples en mouvement : la mobilité spatiale des populations, Sedes, 1986.

Tzvetan TODOROv, Nous et les autres. La réflexion française sur la diversité humaine, Seuil, 1989.

Jean-Didier URBAIN, L'Idiot du voyage : histoires de touristes, Plon, |991.

Thorstein Veblen, The Theory of the Leisure Class, Oxford University Press, 2009 (I re édition 1899).

Philippe VIALLON, "La Méditerranée au risque de la communication touristique", dans Fabienne BAIDER, Marcel BURGER et

Dyonisis Goutsos, La Communication touristique, L'Harmattan, 2004. 